REVISTA INNOVA ITFIP, 7 (1). 110-124 DIC. 2020

\title{
APLICACIÓN DE PMBOK® A LA GESTIÓN DE LA DOCENCIA EN LA UNIVERSIDAD
}

\section{PMBOK® APPLICATION MANAGEMENT OF TEACHING AT THE UNIVERSITY}

\author{
Miguel Arturo Valle Peláez ${ }^{1}$, Andrés David Epifanía Huerta ${ }^{2}$ \\ Valle Peláez, M. A., \& Epifanía Huerta, A. D. (2020). Aplicación de pmbok® a la gestión de la \\ docencia en la universidad. Revista Innova ITFIP, 7(1), 110-124. Recuperado a partir de \\ http://revistainnovaitfip.com/index.php/innovajournal/article/view/112
}

Recibido: Agosto de 2020. Aprobado: Diciembre de 2020

\section{Resumen}

El presente proyecto de investigación está basado en la estandarización del proceso de gestión de la docencia que consisten en adaptar las buenas prácticas del manejo de un proyecto a la organización utilizando los principales procesos del PMBOK®.

Para lo cual, se ha elaborado una Guía de soporte a la gestión de la docencia y se han diseñado un conjunto de materiales para facilitar la implantación de los diferentes procesos establecidos del PMBOK. Además, se ha desarrollado una página web en la nube para que sirva tanto en el compartir los recursos que serán generados, además servirá para recoger las experiencias y sugerencias de los docentes y retroalimentar todo el ciclo para su mejora continua, estandarizando la gestión de la docencia y recoja las buenas prácticas y sugerencia por cada uno de los interesados en mejora del modelo educativo de la Escuela de Ingeniería Informática y de Sistemas de la Universidad San Pedro Chimbote.

Palabras clave: PMBOK®, Gestión de la docencia, Gerencia de Proyectos

\begin{abstract}
This research project is based on the standardization of the management process of teaching consisting of adapting good practices for managing a project for the organization using the main processes of the PMBOK.

For which it has developed a guide support to the management of teaching and have designed a set of materials to facilitate the implementation of various processes established PMBOK. It has also developed a website in the cloud to serve both the sharing of resources to be generated it will also serve to gather the experiences and suggestions of teachers and feedback throughout the cycle for

\footnotetext{
${ }^{1}$ Docente Universitario, Ingeniero de Computación e Informática, / Universidad San Pedro, Perú / ID ORCID 00000003-2255-0938, miguelv_pelaez@hotmail.com

2 Docente Universitario, Magister en Ingeniería de Sistemas / Universidad ULADECH Católica, Perú / ID ORCID 00000002-6643-1829, aepifaniah@uladech.edu.pe
} 
continuous improvement, standardizing management teaching and collect best practices and suggestions for each of those interested in improving the educational model of the School of Computer Science and Systems at the University of San Pedro Chimbote.

Keywords: PMBOK®, teaching Management, Project Management

\section{Introducción}

La gestión de proyectos es una disciplina que integra diversas áreas de aplicación como la ingeniería, administración, economía, política, entre otras. Su interpretación y posterior ejecución es una tarea que demanda gran complejidad debido a la tecnicidad y alto número de sus procesos, el director de un proyecto de software y su equipo de apoyo debe tener no solo la experiencia en el campo sino el conocimiento pertinente para aplicar cualquiera de las metodologías que esta disciplina demanda para ser aplicada de la mejor manera (Jurado Muñoz, 2012) .

La Guía del PMBOK®, desarrollada por el Project Management Instituto, contiene una descripción general de los fundamentos de la Gestión de Proyectos reconocidos como buenas prácticas. Actualmente en su quinta edición, es el único estándar Instituto Nacional Estadounidense de Estándares (ANSI) para la gestión de proyectos. Todos los programas educativos y certificaciones brindadas por el Industria de la Gerencia de Proyectos (PMI) están estrechamente relacionados con el PMBOK.

Para los investigadores que se centran en la gestión de proyectos es la aplicación de los conceptos de gestión del conocimiento como una forma de mejorar el éxito del proyecto (Consilting, 2012).

El trabajo realizado y los resultados obtenidos en un proyecto de innovación docente. El proyecto ha consistido en aplicar las buenas prácticas incluidas en el estándar PMBOK® a la organización e impartición de una asignatura en un entorno universitario. El método desarrollado y los materiales de soporte a la gestión docente, pueden ser de utilidad para cualquier asignatura, independientemente de los estudios, ciclo o curso a los cuales pertenezca, o de la materia que cubra.

Con este proyecto se pretende que el personal docente e investigador tome consciencia de la necesidad de gestionar adecuadamente sus asignaturas, no únicamente de impartirlas con el mayor rigor posible. Disponer de una buena planificación de los contenidos de la materia y del cronograma del curso, no redunda únicamente en beneficio del alumno, sino también del profesor y de la institución que lo alberga. Así, y en el caso de que se haya producido alguna desviación, si el profesor ha tomado buena nota de ello, ésta información podrá ser utilizada para refinar la planificación del próximo curso y actuar en consecuencia. (Mas, Mesquida, A, Gilabert, J., 2012) "Es por esto, que se necesita restructurar la educación para que se adapte a los nuevos requerimientos (...) por lo tanto, el docente deberá gestionar el desarrollo de capacidades que le permitan al estudiante acoplarse a los avances tecnológicos" (Díaz, et al., 2018, p. 151). Buscando" 
REVISTA INNOVA ITFIP, 7 (1). 110-124 DIC. 2020

mejorar la infraestructura educativa con aulas modernas, masificación de la conectividad, uso y apropiación de las tecnologías" (Caycedo, et al., 2019, P.79). Es allí, donde se demuestra la importancia del rol que desempeña el quehacer docente en la generación espacios que promuevan estos desarrollos innovadores en el estudiante de tal forma que les permita afrontar las realidades que le ofrece el entorno" (Díaz, et al., 2018, p. 33); por consiguiente, "Al ser la educación virtual más accesible a todos los usuarios y contar con la posibilidad de acceso a recursos virtuales, sin importar el lugar o condición social, se logra una mayor democratización de la educación" (Palma, et al., 2019, p. 46).

Por lo anteriormente expresado, "es necesario que el líder docente esté abierto a la utilización de nuevas herramientas que fomenten y consoliden un nuevo modo de aprender". (Castrejón \& Peña, 2019, p. 87); es por ello, que estos instrumentos virtuales son tan importantes para todos los usuarios. (Ceballos, Mejía \& Arango 2019)

Finalmente, nos proponemos estandarizar los procesos de gestión educativa en la Escuela Profesional de Ingeniería Informática y de Sistemas, Seguridad Informática e Inteligencia Artificial (EAPIIYS) Universidad San Pedro bajo el marco de referencia a la gerencia de proyectos PMBOK®.

Una importante meta es, elaborar una guía de soporte a la gestión docente y se han diseñado un conjunto de materiales para facilitar la implantación de los diferentes procesos establecidos. Además, se ha desarrollado un portal web para compartir los recursos generados, recoger las experiencias y sugerencias del profesorado y retroalimentar todo el ciclo para su mejora continua en la Universidad San Pedro que requieren de un eficiente manejo y distribución de la información. Es por ello, que es necesaria la Implementación de una metodología basada en Gestión de Proyecto, que permita de una manera ventajosa tener una documentación organizada, reduciendo tiempo y costos.

Asimismo, el presente estudio, tiene como finalidad principal la "Aplicación de PMBOK® a la gestión de la docencia en la Seguridad Informática e Inteligencia Artificial (EAPIIYS) Universidad San Pedro, Chimbote" para estandarizar sus procesos.

\section{Marco Teórico}

\section{¿Qué es el PMBOK®?}

PMBOK son las siglas de Project Management Body of Knowledge, o en español, Guía de los Fundamentos para la Gestión de Proyectos. Esta guía es un instrumento que desarrolló el Project Management Institute (PMI) en la que se establece un criterio de buenas prácticas con relación a la gestión, administración y dirección de proyectos a través de la implementación de técnicas y herramientas que permiten identificar un conjunto de 47 procesos, distribuidos a su turno en 5 macroprocesos generales; esta guía se encuentra en una constante actualización en la cual se le asignan las nuevas tendencias y buenas prácticas en Gestión y Dirección de Proyectos. El PMBOK es la base para poder obtener dos tipos de certificaciones profesionales a nivel 
internacional en el ámbito de la gestión de proyectos: la certificación Profesional en Dirección de Proyectos (PMP) y la certificación Modelo de valoración de activos financieros (CAPM). Actualmente esta Guía se encuentra en su sexta edición, la cual empezó a regir desde el 2018 (Velasco, 2019).

En la sexta edición de PMBOK podemos encontrar la gestión de la calidad, adquisiciones, recursos, riesgos, comunicaciones y la gestión de los interesados del proyecto, esto con el fin de lograr identificar a personas, grupos u organizaciones que de algún modo puedan afectar o por el contrario, ser afectados por un proyecto, para que de este modo se puedan analizar sus expectativas e impacto y en base a eso comenzar a desarrollar nuevas estrategias de gestión adecuadas logrando su participación (Ilarregui, 2018).

\section{El impacto del PMBOK ® en proyectos tecnológicos.}

La sexta edición de la Guía PMBOK. Su importancia del PMBOK y su influencia en los proyectos, ayuda a todos los encargados de gestionar proyectos a tomar mejores decisiones y brindar resultados óptimos, que brinda a las organizaciones un conjunto de procesos, modelos y aspectos favorables para la dirección de proyectos. (Conexión-Esan, 2018)

En respuesta a ello, el PMBOK es de vital importancia en cada proyecto tecnológico para llevar una estructura coherente y eficaz en el logro de cada uno de sus objetivos. Recoge las mejores prácticas y las experiencias que han sido realizadas por administradores de proyectos de las cuales esto se ha conformado por grupos de integración, alcance, tiempo, calidad, costos, recursos humanos, comunicación, que son el impacto en los cambios, métodos, que se generan para asegurar que estos procesos deben estar integrados y asegurar el éxito del diseño y desarrollo de un proyecto. En todo proyecto tecnológico es necesaria las herramientas de PMBOK, porque con esta herramienta indica lo que puede hacerse, más no el cómo, razón por la cual requiere de destreza y habilidades (Sparano Rada, 2008).

\section{La influencia del PMBOK® en la docencia.}

La Guía del PMBOK® es importante en la docencia porque provee un marco de referencia formal para desarrollar proyectos; porque permite guiar y orientar a quienes tienen a su cargo proyectos acerca de la forma de avanzar en los mismos y los pasos que deben seguir necesarios para alcanzar los resultados y objetivos propuestos.

El PMBOK® no indica cómo hacer las cosas. Su importancia radica en que se basa en estándares de calidad a nivel internacional. Al seguirlos, se garantizan los resultados óptimos y una gestión eficaz de cualquier tipo de proyecto en una empresa. Incluye más de 40 procesos que ayudan a las compañías a tomar las decisiones adecuadas, implementar los cambios correctos y planificar con un orden mucho mayor cada uno de sus procedimientos (Consilting, 2012).

Estas son universales y necesarias para los profesionales encargados de la dirección de proyectos. Además, engloba cinco macroprocesos que influyen directamente en la realización de los trabajos:

- $\quad$ El inicio (definir los objetivos principales)

- $\quad$ La planificación (cómo se desarrollarán las metas)

- La ejecución (las estrategias para lograrlo)

- $\quad$ El control (supervisión y monitoreo) 
REVISTA INNOVA ITFIP, 7 (1). 110-124 DIC. 2020

- $\quad$ El cierre (aceptación, retroalimentación y satisfacción) (Consilting, 2012).

\section{Materiales y métodos}

Para las fases del proyecto de inicio es regulada por la por normativas y estándares de acreditación que son propias de la universidad y escuela Ingeniería Informática y de Sistemas de la Universidad San Pedro.

Del proyecto de investigación científica busca estandarizar las Fases de proyectos de planificación, ejecución, seguimiento y cierre para los procesos de gestión educativa en la EAPIIYS - USP aplicando el desarrollo de sus 47 procesos a las áreas del conocimiento utilizando el marco de referencia de la guía de las buenas prácticas de proyecto estandarizando un modelo de mejora continua Internacional.

Tabla 1: Ciclo de Vida del docente por semestre Académico Seguridad Informática e Inteligencia Artificial (EAPIIYS) Universidad San Pedro (USP)

\begin{tabular}{|c|c|c|c|c|}
\hline \multirow{2}{*}{$\begin{array}{l}\text { Áreas } \\
\text { Conocimiento }\end{array}$} & UNIVERSIDAD & \multicolumn{3}{|c|}{ CATEFRATICO } \\
\hline & Inicio & Planificación & Ejecución & $\begin{array}{l}\text { Seguimientoy } \\
\text { Control }\end{array}$ \\
\hline Gestión de la integración & $\begin{array}{l}\text { Desarrollo el acta de constitución } \\
\text { Del proyecto }\end{array}$ & $\begin{array}{l}\text { Desarrollar el plan para la dirección de } \\
\text { proyectos }\end{array}$ & $\begin{array}{l}\text { Dirigir, gestionar la ejecución del } \\
\text { proyecto }\end{array}$ & $\begin{array}{l}\text { Monitorear el control del trabajo del proyecto } \\
\text { Realizar el control integrado de cambios }\end{array}$ \\
\hline Gestión del Alcance del Pr. & Recopilar requisitos & $\begin{array}{l}\text { Definir el alcance } \\
\text { Crear la EDT }\end{array}$ & & $\begin{array}{l}\text { Verificar el alcance } \\
\text { Controlar el alcance }\end{array}$ \\
\hline Gestión del Tiempo del Pr. & Estimar el cronograma académico & $\begin{array}{l}\text { Definir las actividades } \\
\text { Secuenciarlas } \\
\text { Estimar los recursos } \\
\text { Estimar la duración } \\
\text { Desarrollar el cronograma }\end{array}$ & & Controlar el cronograma \\
\hline Gestión de los Costos del Pr. & $\begin{array}{l}\text { Estimar los costos determinar el presupuesto plan } \\
\text { operativo, y estratégico }\end{array}$ & & & \\
\hline Gestión de la Calidad del Pr. & $\begin{array}{l}\text { Planificar l c calidad } \\
\text { Aseguramiento de la calidadControl de la calidad }\end{array}$ & Planificar la calidad & Aseguramiento de la calidad & Control de la calidad \\
\hline $\begin{array}{l}\text { Gestión de los Recursos } \\
\text { Humanos de Pr. }\end{array}$ & $\begin{array}{l}\text { Desarrollar el plan de recursos humanos. } \\
\text { Adquirir el equipo del proyecto } \\
\text { Desarrollar el equipo del proyecto } \\
\text { Dirigir el equipo del proyecto }\end{array}$ & Auto capacitación docente & & \\
\hline $\begin{array}{l}\text { Gestión de las Comunicaciones } \\
\text { del Pr. }\end{array}$ & Diseñar el plan de comunicaciones & Planifica las comunicación & $\begin{array}{l}\text { Gestionar la expectativa de los } \\
\text { interesados }\end{array}$ & Informar el desempeño \\
\hline Gestión de Riesgos del Pr. & $\begin{array}{l}\text { Planificar la gestión de riesgos } \\
\text { Identificar los riesgos } \\
\text { Análisis cualitativo } \\
\text { Análisis Cuantitativo } \\
\text { Monitorizar y controlar los riesgos } \\
\text { Planificar la respuesta a los riesgos }\end{array}$ & $\begin{array}{l}\text { Planificar la gestión de riesgos } \\
\text { Identificar los riesgos } \\
\text { Análisis cualitativo } \\
\text { Análisis Cuantitativo }\end{array}$ & & Monitorizar y controlar los riesgos \\
\hline $\begin{array}{l}\text { Gestión de la Adquisiciones del } \\
\text { Pr. }\end{array}$ & $\begin{array}{l}\text { Planificar las adquisiciones } \\
\text { Efectuar las adquisiciones } \\
\text { Administrar las adquisiciones } \\
\text { Cerrar las adquisiciones } \\
\end{array}$ & Planificar las Adquisiciones & & \\
\hline $\begin{array}{l}\text { Gestión de la Lista de } \\
\text { interesados }\end{array}$ & Identificar a los interessados & Planificar la gestión de los interesados & $\begin{array}{l}\text { Distribuir la información a los } \\
\text { interesados }\end{array}$ & control el compromiso de los interesado \\
\hline
\end{tabular}

Fuente: Elaboración propia 


\section{Resultados}

Elaboración de la guía de PMBOK®

Tabla 2: Acta Constitución de proyecto

\section{ACTA DE CONSTITUCIÓN DEL PROYECTO CÓDIGO MAVP001 - versión 5}

\begin{tabular}{|l|l|l|l|l|l|}
\hline PROYECTO & Mejora del modelo Educativo en la Universidad San Pedro \\
\hline PATROCINADOR & EAPIIYS & \multicolumn{5}{|l|}{$\mid$} \\
\hline PREPARADO POR: & Ing. Miguel Arturo Valle Pelaez, & FECHA & 06 & 01 & 2015 \\
\hline APROBADO POR: & Director EPAIIYS: Ing Marle P, Dr & FECHA & 15 & 12 & 2015 \\
\hline
\end{tabular}

El producto del proyecto es la mejora del modelo educativo de la Universidad San Pedro.

1. Estandarización del modelo educativo con los sílabos en todas las filiales de la carrera profesional

2. Rediseño del plan curricular de la carrera profesional verano 2015

3. Rediseño del plan estratégico de la facultad 2016

4. Diseño de los instrumentos de evaluación docente

5. Diseño de un plan de capacitación a los docentes de la universidad

6. Elaboración de un mecanismo de seguimiento y bolsa de trabajo a los practicantes y egresados

7. Diseñar un plan de Participación en Proyección Social para docentes y alumnado de la EAPIIYS

8. Revista electrónica de proyectos de investigación

9. Programas de bienestar universitario

10. Programa de soporte a la tecnología verde

11. Programas de Capacitación: Pregrado, Docente, egresados, Comunidad.

\section{OBJETIVOS ESTRATÉGICOS USP}

Impulsar la investigación científica como el resultado de proyectos de innovación tecnológica para que la EAPIIYS contribuya con el desarrollo de la Región y el País

\section{PROPÓSITO DEL PROYECTO}

Mejorar el modelo educativo de la USP para apoyar a la acreditación de la escuela de Ingeniería Informática y de Sistemas y elevar la calidad de enseñanza aprendizaje.

\section{OBJETIVOS DEL PROYECTO}

- Implementar un nuevo modelo educativo en la USP para la escuela EAPIIYS

- Llevar a cabo la implementación con presupuesto de la EAPIIYS.

- La implementación deberá incluir en un plazo máximo de 1 año

- La implementación con la metodología PMBOK® que está recomendada por el PMI para la gestión de proyectos y de la metodología COBIT.

- Realizar la ejecución del proyecto cumpliendo con las especificaciones brindadas por el informe de Estándares de Calidad brindado por el SINEACE

4. FASES DEL PROYECTO 5. PRINCIPALES ENTREGABLES 


\begin{tabular}{|c|c|}
\hline & $\begin{array}{l}\text { - Plan estratégico } \\
\text { - Plan curricular } \\
\text { - Plan de estudios } \\
\text { - Plan de capacitación docente } \\
\text { - Diseño de instrumentos de evaluación } \\
\text { docente } \\
\text { - Diseño de modelo de gestión de } \\
\text { investigación } \\
\text { - Diseño de programa de bienestar } \\
\text { universitario }\end{array}$ \\
\hline Construir, adquirir e implementar & 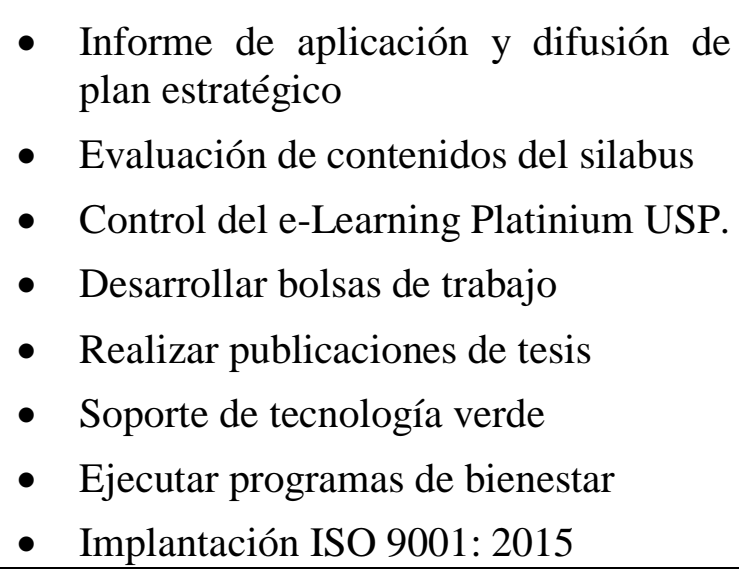 \\
\hline Evaluar, dirigir y monitorear & $\begin{array}{l}\text { - Evaluar resultados plan curricular } \\
\text { - } \text { Aplicar evaluación docente } \\
\text { - Evaluar cumplimiento de silabus } \\
\text { - Evaluar metodología de enseñanza } \\
\text { - } \quad \text { Propaluar avances de acreditación } \\
\end{array}$ \\
\hline Entregar, servir y dar soporte & $\begin{array}{l}\text { - Formación de inventario de } \\
\text { conocimientos }\end{array}$ \\
\hline
\end{tabular}

Fuente: Elaboración propia 
Figura N1. Gestión de alcance Proyecto de mejora Educativa USP

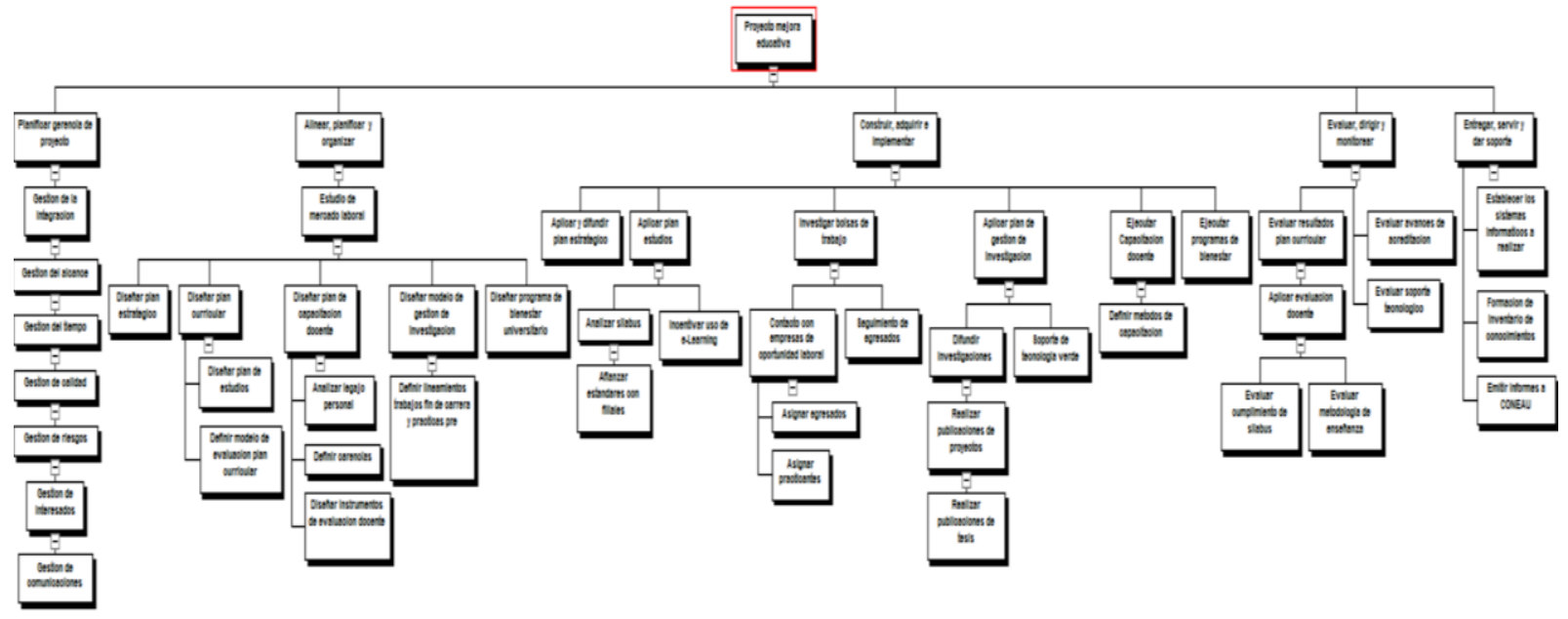

Fuente: Página ecorfan.org - Proyectos de Gestión Educativa

\section{Herramientas de calidad:}

Figura N2. Mejora del plan de estudios

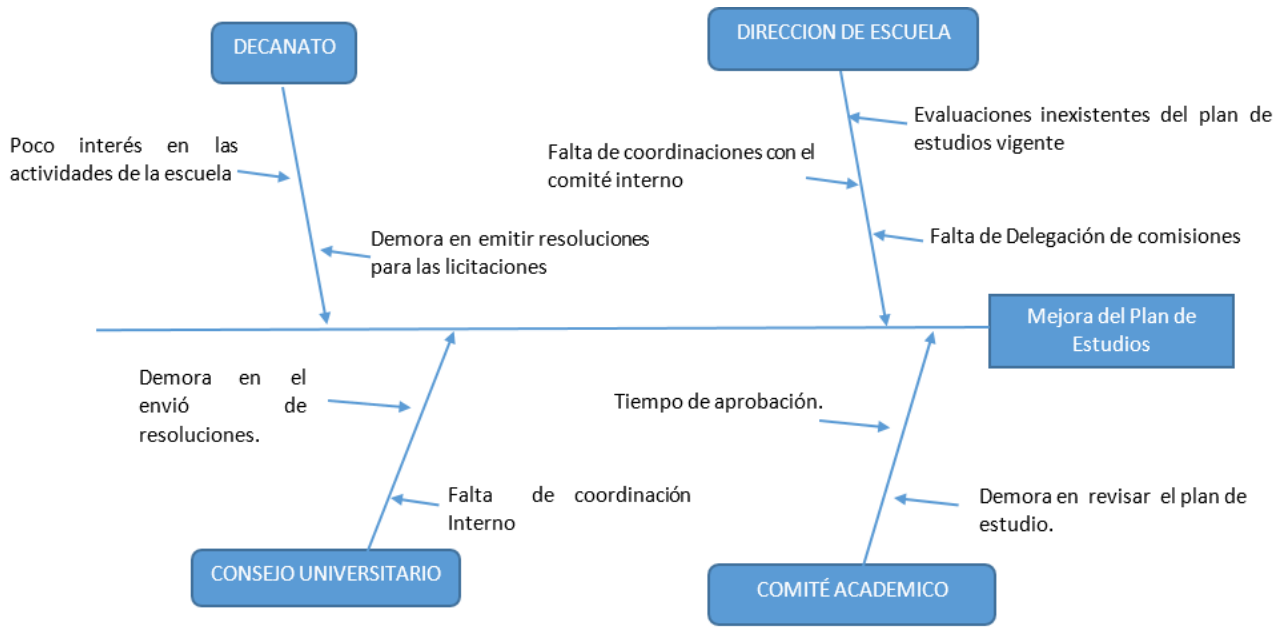

Fuente: Elaboración propia mediante el diagrama de Ishikawa 
REVISTA INNOVA ITFIP, 7 (1). 110-124 DIC. 2020

Figura N3. Gestión Educativa evaluación y elaboración plan de estudios

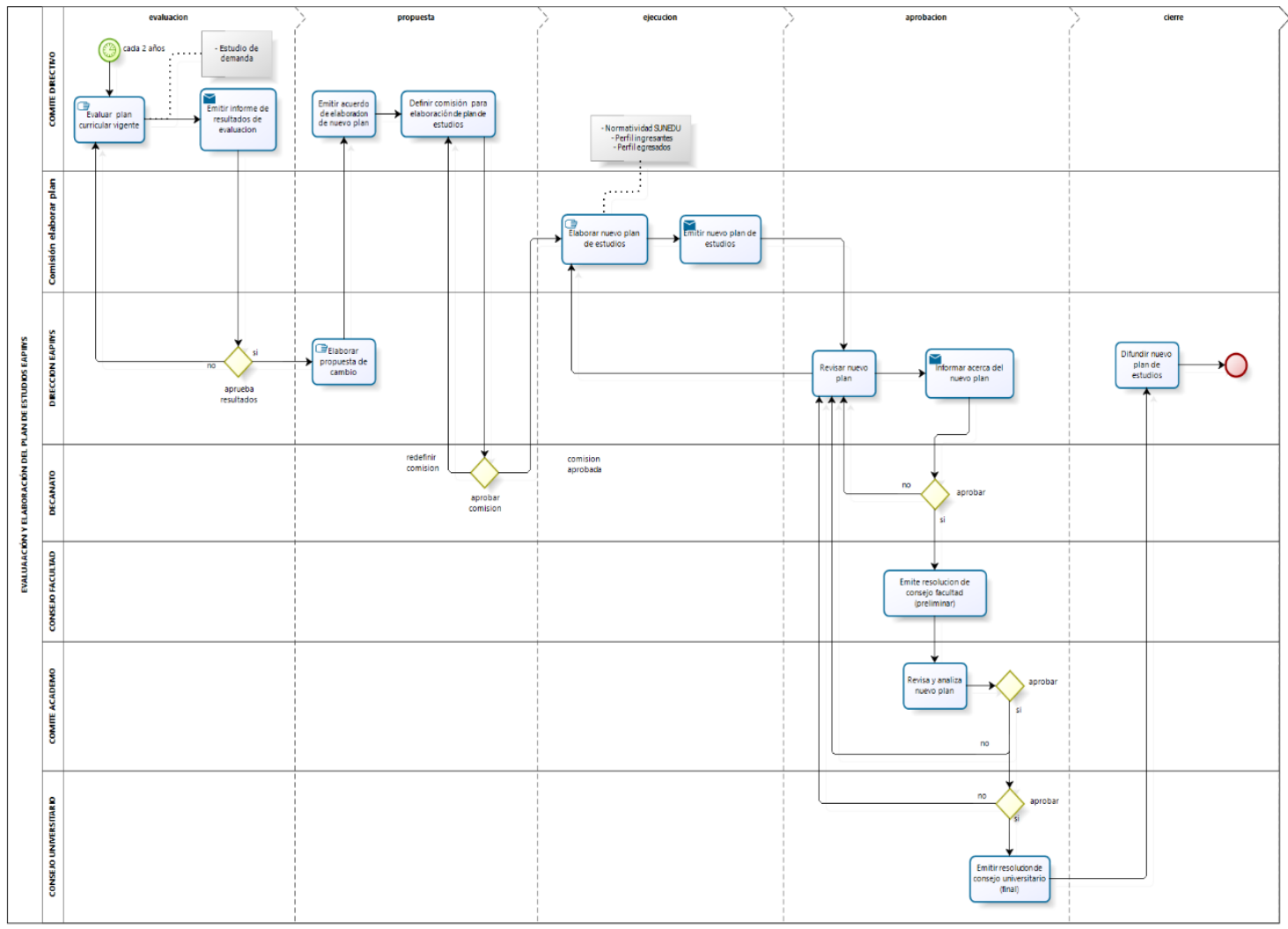

Fuente: Elaboración propia mediante el modelador de procesos Bizagi 
Figura N4. Procesos de evaluación del Egresado USP

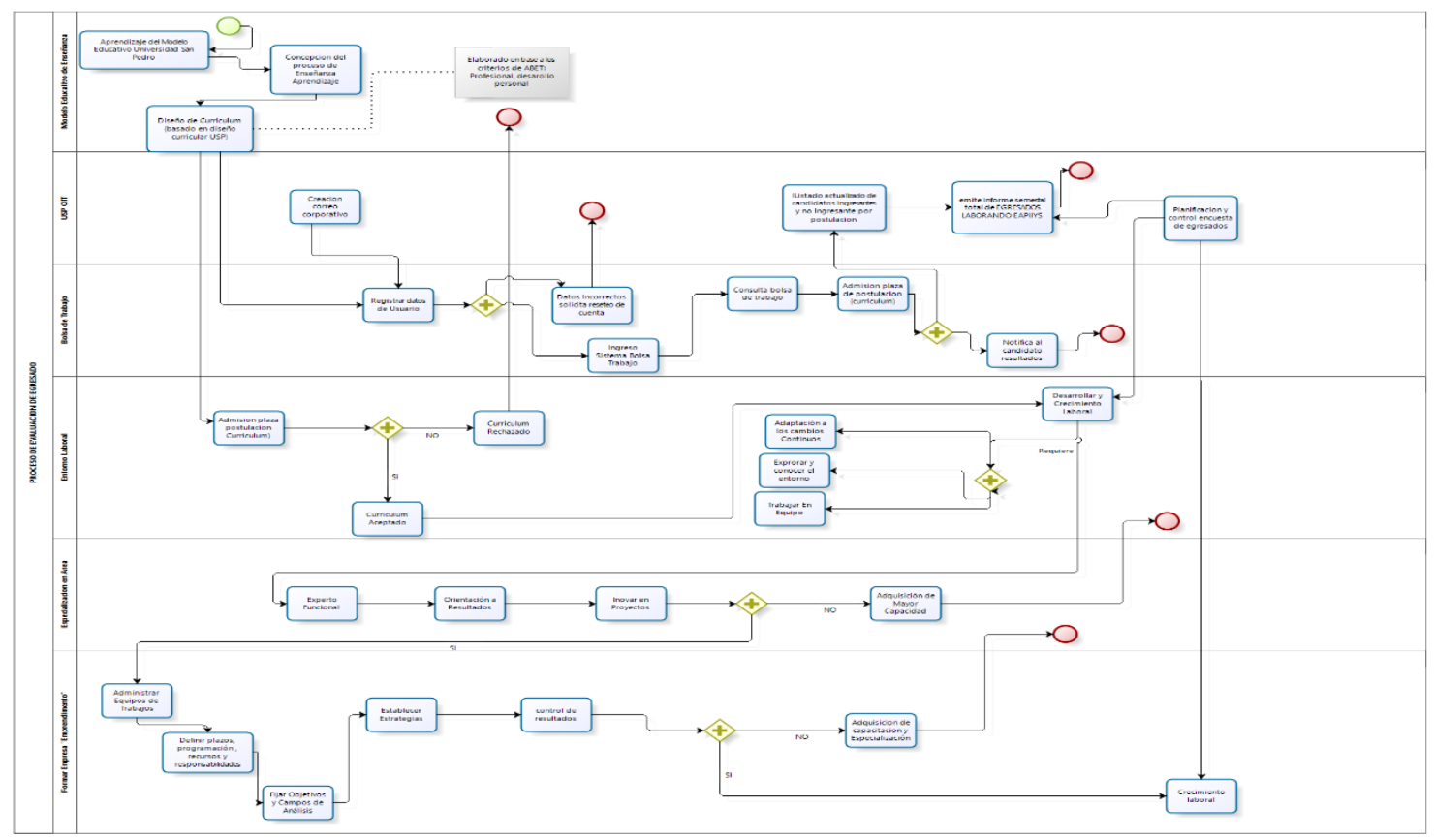

Fuente: Elaboración propia mediante el modelador de procesos Bizagi

\section{Análisis y discusión}

El presente trabajo de investigación propuso aplicar el método de gestión basado en el PMBOK en el proceso de desarrollo de la docencia en la Universidad, como además cabe destacar que según la autora Ilarregui L. (2018), quien escribió las buenas prácticas de la gestión de proyectos de la guía PMBOK a la gestión del desarrollo de las asignaturas de los docentes, evidenciándose como resultados que el método desarrollado y los materiales de soporte fueron de utilidad para la gestión de las asignaturas con el objetivo de permitir a los docentes cumplir mediante las directrices recomendadas al PMI.

En el resultado culminado se obtuvieron las evidencias que el método de gestión propuesto brindo las herramientas que mejorarían la gestión del proceso de desarrollo de investigación con el fin a la acreditación de la escuela de Ingeniería Informática y de Sistemas en destacar la calidad de aprendizaje, además teniendo similitud con los resultados, y se aplicó el PMBOK para cumplir con las directrices establecidas, en el caso de este estudio fue recomendadas por la Sistema Nacional de Evaluación, Acreditación y Certificación de la Calidad Educativa (SINEACE), que aceptara en permitir los directores de investigación a la universidad para el cumplimiento de los procesos y paso a desarrollar la gestión brindados por el método.

Cabe destacar que según el autor (Sparano Rada, 2008), realizó la continuidad del estudio referido y lograron construir un entorno personal para contribuir con la gestión de los docentes con el desarrollo de las asignaturas, que también fue integrado herramientas que apoyarían al logro de muchas actividades propuestas, para determinar el método aplicado con un número 
REVISTA INNOVA ITFIP, 7 (1). 110-124 DIC. 2020

mayor de asignaturas. Además en la investigación no se construyó un entorno personal porque no logra responder a los criterios de estudios, pero también, se elaboró un portal web como un apoyo y herramienta para este método, donde se haya documentos, entre otros formatos que es referencia a la gestión de la investigación, con la finalidad de no afectar la planificación de la tareas y respetando los distintos formatos en el estudio; para culminar sobre este aporte conlleva que los recursos humanos desarrollen la productividad, quiere decir que permitirá minorar el tiempo de búsqueda, como el acceso y elaboración de archivos o formatos que validen la calidad de la gestión de los procesos y procedimientos.

Hay otros trabajos desarrollados según el autor Barato (2015) demostró que la aplicación del PMBOK a la gestión de riesgos y proyectos de investigación académica, el primero ayudó a alinear la gestión de riesgos con las especificidades del desarrollo de la investigación científica, el segundo condujo al desarrollo de un marco de gestión de proyectos PMBOK, los resultados son similares a los del estudio ya que ambos casos han sido contextualizados en las áreas de conocimiento del PMBOK. Sin embargo, el estudio solo consideró procesos y procedimientos que corresponden a los estándares de investigación propuestos por Sistema Nacional de Evaluación, Acreditación y Certificación de la Calidad Educativa (SINEACE).

Así mismo, estudios realizados según el autor Bucero Torres (2013), ha demostrado que la adaptación de la guía del PMBOK se puede aplicar a cualquier proceso de autoevaluación conducente a la acreditación, de la misma forma que el estudio utilizó el método PMBOK para facilitar la gestión del desarrollo de la investigación, que si bien esto es cierto, estaba alineado con un área específica, pero ayuda a brindar insumos para lograr el cumplimiento de los estándares de calidad, a lo cual se le ha realizado un tratamiento tomando en cuenta los procesos y procedimientos de los niveles estratégico, táctico y operativo que caracterizan la gestión.

Del proyecto se desarrolló la guía de las buenas prácticas para estandarizar la gestión educativa:

- Ciclo de Vida del docente por semestre Académico en la Seguridad Informática e Inteligencia Artificial (EAPIIYS) Universidad San Pedro (USP).

- Fases del Proyectos, Proyecto, actividades e indicadores de evaluación modelo de gestión educativa USP

- Gestión de la Integración: Características, funcionalidades, soporte entre otros del producto o servicios, alineamientos, objetivos factores críticos de éxito, fases y sus principales entregables, interesados clave

- Gestión de alcance las fases del modelo de gestión educativa

- Gestión del tiempo por el control de su sílabo de clases al iniciar ciclo subido a plataforma y el control por semana, las herramientas, los medios que hace uso y requerimiento para las mejores prácticas del curso dictado

- Gestión de calidad: metodologías propuesta para el uso de mejora de los procesos académicos 
- Matriz de calidad: estándares, actividades de prevención, mitigación y desarrollo de controles

- Herramientas de calidad Ishikawa para ver los problemas su causa y origen

- Como toda base de la educación es su plan de estudios conocer los procesos que rigen el control de la calidad.

Por lo tanto, se estandariza los procesos para mejora el modelo de gestión educativa en la Seguridad Informática e Inteligencia Artificial (EAPIIYS) y obtener la calidad de la formación continua de nuestros alumnos y egresados. promoviendo la producción de investigación e Impulsar una cultura de responsabilidad social dentro y fuera del País.

\section{Conclusiones}

El método desarrollado y los materiales de soporte a la gestión de la docencia, pueden ser de utilidad para cualquier asignatura, independientemente de los estudios, ciclo o curso a los cuales pertenezca, o de la materia que cubra.

Está previsto iniciar un proyecto piloto en el que participen algunos de los profesores de nuestra Escuela. Los participantes seguirán la Guía elaborada y utilizarán los materiales generados que se encuentran a su disposición en el portal web de soporte al proyecto. Si este proyecto piloto funciona bien a nivel interno, se espera poder reproducir la experiencia pero ya a nivel de toda la escuela, incorporando a todos los profesores que imparten materias con requisitos y necesidades diferentes.

Utilizando las buenas prácticas de gestión de proyectos es posible incidir sobre muchos más aspectos del ciclo de vida de una asignatura, de los que se han considerado durante esta investigación. Tomando como ejemplo el modelo académico propuesto por la Universidad San Pedro controlado por el director de escuela, este es necesario para impartir cada materia de un plan de estudios. En este Plan, se identificarían las habilidades requeridas para impartir la materia con garantías de calidad, se incluiría la identificación de las necesidades de capacitación para el profesorado y se documentarían los diferentes roles y responsabilidades posibles. Este resultado podría ser de gran utilidad

\section{Referencias Bibliográficas}

Bustamante Romero J. (2015). Método de gestión basado en el PMBOK para el proceso de desarrollo de investigación de las carreras universitarias. Recuperado a partir de: https://alicia.concytec.gob.pe/vufind/Record/USMP_93a99597d1bc752fd793882375b57 $\underline{\text { ee4/Details }}$ 
REVISTA INNOVA ITFIP, 7 (1). 110-124 DIC. 2020

Borroto P. (2006), gerencia de los proyectos educativos integrales (PEI). algunas indicaciones para su perfeccionamiento.

Recuperado el 14 de mayo del 2015 desde:

http://web.a.ebscohost.com/ehost/detail/detail?sid=3e183e98-44f2-4013-864e4ad3e227232d\%40sessionmgr4003\&vid=0\&hid=4212\&bdata=Jmxhbmc9ZXM\%3d\#db $=$ fua $\& A N=25383001$.

Barato J. Guía de estudio en español para la capacitación del Director de Proyectos. Preparación para el Examen PMP/CAPM del PMI según la Guía del PMBOK. [Online].; 2015.

Recuperado de:

https://books.google.com.pe/books?id=JiW-

DgAAQBAJ\&dq=PMBOK\%C2\%AE+a+la+gesti\%C3\%B3n+de+la+docencia+en+la+U niversidad\&source $=\mathrm{gbs}$ navlinks_s

Bucero Torres A. La dirección de proyectos: Una nueva visión. [Online].; 2013.

Recuperado de:

https://books.google.com.pe/books?id=ERYBAQAAQBAJ\&dq=PMBOK\%C2\%AE+a+ $\underline{\text { la+gesti } \% \text { C3\%B3n+de+la+docencia+en+la+Universidad\&source=gbs_navlinks_s }}$

Castrejón Reyes, V. \& Peña Estrada, C. (2019). Liderazgo docente una oportunidad para afrontar los desafíos en el aprendizaje digital. Revista Innova ITFIP, 4(1), 84 - 94. Recuperado a partir de http://www.revistainnovaitfip.com/index.php/innovajournal/article/view/72

Caycedo, M., Palma, E., Ortiz, I., Garrido, F., Rondón, A. \& Guzmán, R. (2019) El desarrollo territorial desde la caracterización de la comuna $\mathrm{N}^{\circ} 1$ del municipio de Ibagué - TOLIMA. Edición 1. Espinal Tolima. Instituto Tolimense de Formación Técnica Profesional-ITFIP. 106 p. ISBN: 978-958-52015-5-2

Ceballos, O. I., Mejía Castellanos, L. A., \& Arango Medina, D. (2019). Auditoria de usabilidad de herramientas implementadas en plataformas virtuales para ofertar servicios con responsabilidad social. Revista Innova ITFIP, 5(1), 64-77. Recuperado a partir de http://www.revistainnovaitfip.com/index.php/innovajournal/article/view/56

Díaz, M., Palma, E., Leiva, F., Varón O., Alarcón, A. y Sandoval, L. (2018) “Avances de investigación en ciencias económicas, administrativas y contables" Editorial: Instituto Tolimense de Formación Técnica Profesional-ITFIP 187 p. ISBN: 978-958-59986-4-3.

Díaz, M., Palma, E., Leiva, F., Moreno, L., Caycedo. M. \& Guzmán, R. (2018) La Investigación como Aporte a la Generación del Conocimiento en las Ciencias Económicas, Administrativas Y Contables Editorial: Instituto Tolimense de Formación Técnica Profesional-ITFIP 60 p. ISBN: 978-958-59986-5-0. 
Conexión-Esan (2018) La importancia del PMBOK y su influencia en un proyecto. Recuperado de: https://www.esan.edu.pe/apuntes-empresariales/2018/10/la-importancia-del-pmbok-ysu-influencia-en-un-proyecto/

Guía del PMBOK® - (2008) Gestión_Dharma Consulting. Cuarta Edición Recuperado de: https://es.slideshare.net/Dharmacon/pi-013-01

Ilarregui L. (2018) EspacioBim. [Online].

Recuperado de:

https://www.espaciobim.com/pmbok.

Jurado Muñoz, J., Pardo Calvache, C. (2012)

Recuperado el 14 de mayo del 2015 desde:

http://web.a.ebscohost.com/ehost/detail/detail?sid=9a8ddcb2-47b2-4457-9d44-

769ad1288184\%40sessionmgr4004\&vid=0\&hid=4212\&bdata=Jmxhbmc9ZXM\%3d\#db =fua\&AN=95420203.

Mas, Mesquida, A, Gilabert, J., (2012) Aplicación de PMBOK a la gestión de la docencia en universidades en Universitat de les Illes Balears (UIB) Palma de Mallorca, España. Recuperado el 08 de octubre del 2014, desde: http://projectmanagement.uib.es/?lang=es

Palma, E., Escovar, E., Bedoya, D., Garrazza, N., Fenoglio, N., Berizzo, L., Volker, R., Salgado, L., García, J. \& Moreno, L. (2019) La Gestión Investigativa en las Mipymes, una mirada desde Colombia, México y Argentina. Edición 1. Espinal Tolima. Editorial: Instituto Tolimense de Formación Técnica Profesional-ITFIP.136 p. ISBN: 978-958-52015-4-5

Perurena C. (2012) Gestión e innovación en Tecnología Educativa: experiencias en universidad de La Habana, Cuba.

Recuperado el 08 de octubre del 2014 desde:

http://web.a.ebscohost.com/ehost/detail/detail?sid=69ec4974-349d-4ff3-a3762444cd37b1a6\%40sessionmgr4001\&vid=0\&hid=4214\&bdata=Jmxhbmc9ZXM\%3d\#db= fua\&AN=96333132

Reich, B. (2006) SEARCHING FOR KNOWLEDGE IN THE PMBOK GUIDE. Recuperado el 08 de octubre del 2014 desde: http://web.a.ebscohost.com/ehost/detail/detail?sid=e27a5667-816f-4229-90f0- 
REVISTA INNOVA ITFIP, 7 (1). 110-124 DIC. 2020

e31de84869de\%40sessionmgr4001\&vid=0\&hid=4214\&bdata=Jmxhbmc9ZXM\%3d\#db $=$ bth $\& A N=21463500$

Sparano Rada H. (2008) Impacto de las áreas de conocimiento de la administración de proyectos a través del PMBOK.

Recuperado de:

$\underline{\text { https://dialnet.unirioja.es/servlet/articulo?codigo }=3797760}$

Sánchez E. Evaluación Del Erp University De La Universidad Uladech Católica Usando Iso/Iec 15504-4 - Chimbote; 2017. [Online].; 2019.

Recuperado de:

http://repositorio.uladech.edu.pe/bitstream/handle/123456789/13752/EVALUACI\%C3 \%93N_DE_PROCESOS_SANCHEZ_PEREZ_EDGAR_PEDRO.pdf?sequence $=4$ is Allowed=y

Velasco R. (2019) Gestión de Proyectos Plus. [Online]. Recuperado de:

https://gestiondeproyectosplus.com/que-es-pmbok/ 